\title{
Patient-Centered Care: Where Policy Meets Practice
}

\author{
Eve J Higgin botham* \\ Department of Ophthalmology, University of Pennsylvania, USA
}

Submission: March 13, 2017; Published: April 13, 2017

*Corresponding author: Eve J Higginbotham, SM, MD, Professor of Ophthalmology, Vice Dean, Perelman School of Medicine, Senior Fellow, Leonard Davis Institute, University of Pennsylvania, Philadelphia, Pennsylvania, USA, Email: ehig@upenn.edu

\section{Commentary}

A 59-year-old woman is referred to you by her primary care provider (PCP) for a routine eye examination. She had never undergone a comprehensive eye exam previously and was looking forward to the opportunity to have her eyes examined by an ophthalmologist. She purchased reading glasses for the first time 5 years ago, but now even those spectacles were not addressing her visual needs. Her complaints to her PCP prompted the referral. Additional history revealed that this patient was recently laid off and was now insured on Medicaid, which had been expanded in her state following the passage of the Affordable Care Act.[1]

On examination, her visual acuity was $20 / 40$ OD and 20/60 OS. Angles were open OU 360 degrees and IOP measured $25 \mathrm{mmHg}$ OD and $32 \mathrm{OS} \mathrm{mmHg}$. SLE revealed clear corneae, deep and quiet anterior chambers, and $1+$ nuclear sclerosis. Central corneal thickness measurements were 550um OD and 555um OS. Optic nerves evidenced moderate cupping, 0.70D and 0.80S.

Patients such as this 59-year-old woman enter our offices every day. Her visual fields and optic nerve imaging confirmed mild glaucoma OD and moderate glaucoma OS. Her physician set a target pressure of $16-18 \mathrm{mmHg}$ OD and $14-16 \mathrm{mmHg}$ OS. Now that her diagnosis has been established and a plan discussed with the patient, it is clear based on her history shared at the beginning of her examination that the reliance only on topical medication would not be realistic. Although generic medications may be are affordable options, given the list of medications that she manages for her diabetes and hypertension, it is clear that additional options will need to be considered.

This case reminds us of the importance of considering the whole patient rather than solely the patient's ocular presentation. Being realistic in the beginning of a patient's relationship with the provider is critical for establishing the basis for an effective partnership to slow or halt the progression of disease. It is also important to understand the healthcare landscape that shapes patients' choices for coverage of their care. For example, in the
United States, the Affordable Care Act (ACA) that was launched in 2010, expanded Medicaid coverage to previously uninsured patients in more than half of the country [1]. This patient had purchased insurance under the ACA, Now that her insurance may be discontinued, she is concerned that she will be uninsured again.

Given her circumstance, the physician decided to begin treatment with generic latanoprost once daily as a monocular trial in the left eye; the option of selective laser trabeculoplasty (SLT) is also discussed. The patient returned Measuring and her intraocular pressures are essentially unchanged, measuring 22 OD mm Hg and 29 OS mm Hg. The patient admitted not being able to afford the medication and agrees to proceed with SLT. SLT is performed in both eyes and six weeks later IOP measured $18 \mathrm{mHg}$ OD and $20 \mathrm{mmHg}$. Additional SLT treatment is considered to get her pressures closer to her target.

This case brings up three discussion points the author wishes to highlight:

a. The benefits of treatment with SLT

b. The importance of understanding the context of care

c. Why eye health needs to be a population health priority

The benefits of treatment with SLT in early and advanced glaucoma has been reported by others [2,3]. Notably, in a series of 26 eyes with early glaucoma, investigators reported greater than a $20 \%$ reduction in intraocular pressure and a reduction in intraocular pressure less than $21 \mathrm{mmHg}$ in $62.9 \%$ of treated eyes, and with retreatment an additional $7.4 \%$ were noted to evidence a reduction in intraocular pressure. Among 44 eyes with advanced glaucoma, an IOP reduction greater than $30 \%$ reduction in IOP and an IOP less than $18 \mathrm{~mm} \mathrm{Hg}$ was noted in $50 \%$ of eyes treated. These eyes were followed for 1 year [3] When compared to either medications or surgery over a 5-year period, laser trabeculoplasty has been noted to be the most cost effective[4]. Thus, for our patient, given her challenges with the affordability of medications, SLT is a reasonable option. 
In the United States, the landscape for patients who struggle to afford healthcare is currently uncertain, given current efforts to repeal the Affordable Care Act [1]. Thus, understanding the context in which this patient must consider her care is important. Personalizing the treatment plan for patients should consider the realities of the impact of care, in addition to the best, evidence-based options. At 59 years of age, this patient is not yet eligible for Medicare and thus, the cost effectiveness of the laser trabeculoplasty [4] is an important consideration. In addition, the outcome of the proposed treatment is critically important. If the patient can be managed on fewer medications or on no medications, then her quality of life will benefit.

Consider the evidence of the effectiveness of surgical intervention if the patient had presented with angle closure glaucoma. If this patient had presented with angle closure glaucoma, clear lens extraction may have been an option for her, particularly given the results of the EAGLE study. The EAGLE study assessed the effectiveness of clear lens extraction in patients with angle closure glaucoma. In a randomized controlled study design, randomizing patients to either laser iridotomy or surgery, the investigators reported clear lens extraction to be more effective and cost effective than laser iridotomy [5]. Regardless of the outcome of the debate related to the Affordable Care Act, [1] it is likely that as a discipline, it will be important to assemble additional data such as the EAGLE5 and the Cantor evaluation related to laser trabeculoplasty [3] to support the reimbursement of specific clinical decisions, as metrics for reimbursement move from volume to value [6]. Timing for our patient's procedure is important, given the imminent changes in her healthcare coverage.

The last point highlights a recent report from the Institute of Medicine (now named the National Academy of Medicine) released in the fall of 2016, entitled, "Making Eye Health a Population Health Imperative: Vision for Tomorrow" underscores importance of preventing irreversible blindness and suggests strategies for minimizing vision impairment. Nine recommendations are offered addressing public awareness, the need for greater evidence to guide policy, importance of expanding access, enhance the capacity of public health resources, and the need for promoting community action [7]. Our patient represents a missed opportunity for earlier diagnosis and intervention. Encouragement by other providers with whom she may have interfaced even five years prior to her presentation would have given her a greater likelihood of avoiding irreversible loss of vision related to glaucoma. Only by elevating the eye health in the minds of nonophthalmic clinicians, the public, and insurers will the needle significantly move in preventing blindness. Our patient has benefitted from work of many innovators and researchers, her ability to maintain her visual lifeline for the rest of her life rests largely with her as she navigates the healthcare landscape. The provider is her partner in this journey, effectively guiding her with a focus on the context of her care.

\section{References}

1. Obama B (2016) United States Health Care Reform: Progress to Date and Next Steps. JAMA 316(5): 525-532.

2. Zhou Y, Aref AA (2017) A Review of Selective Laser Trabeculoplasty: Recent Findings and Current Perspectives. Ophthalmol Ther doi: 10.1007/s40123-017-0082-x.

3. Schlote T, Kynigopoulos M (2016) Selective laser trabeculoplasty (SLT): 1-year results in early and advanced open angle glaucoma. Int Ophthalmol 36(1): 55-61.

4. Cantor LB, Katz LJ, Cheng JW, Chen E, Tong KB, et al. (2008) Economic evaluation of medication, laser trabeculoplasty and filtering surgeries in treating patients with glaucoma in the US. Current Med Res Opin 24(10): 2905-2918.

5. Azuara-Blanco A, Burr J, Ramsay C, Cooper D, Foster PJ, et al. (2016) Effectiveness of early lens extraction for the treatment of primary angle-closure glaucoma (EAGLE): a randomised controlled trial. Lancet 388(10052): 1389-1397.

6. Cohen CG, Repka M, Hancock R (2017) Medicare's New Quality Payment Program for Physicians. Ophthalmology 124(1): 7-8.

7. Teutsch S, McCoy M, Woodbury B, Annalyn W (2016) Making Eye Health a Population Health Imperative: Vision for Tomorrow. The National Academies Press, Washington, USA.

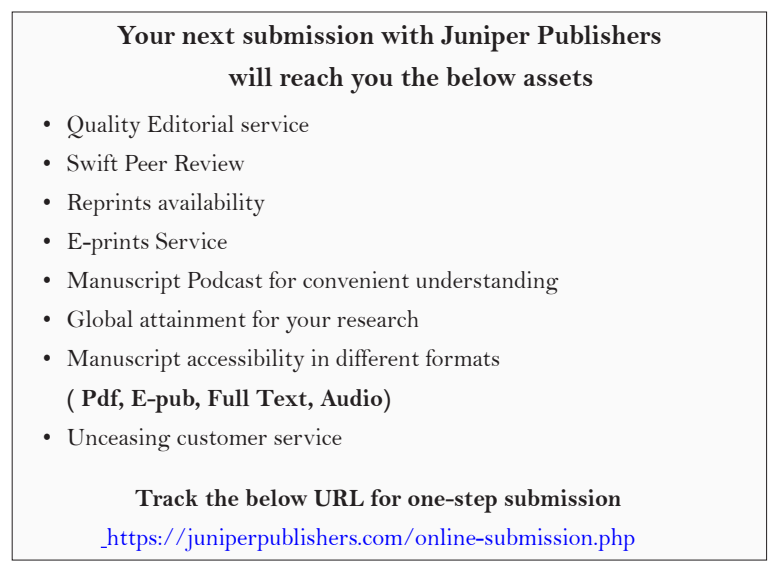

